\section{A rare case of compression atelectasis due to spontaneous diaphragmatic hernia: a case report}

\section{DOI: 10.52629/jamsa.v9i1.203}

Abstract Type IV diaphragmatic hernia is a rare case which has various clinical manifestations. The clinical manifestations as a result of the protruded organ towards thoracic cavity in diaphragmatic hernia vary from gastrointestinal symptoms such as epigastric pain, dysphagia and acid reflux. However, in rare occasions, dyspnea may present as a result of compression atelectasis due to excessive increase in intrathoracic pressure. Here, we report a 57-year-old male patient who had spontaneous diaphragmatic hernia, admitted with dyspnea as a chief complaint. This report aims to add another perspective for physicians in facing patients with atelectasis as a result of a high level of lung compression secondary to type IV diaphragmatic hernia.

Keywords Diaphragmatic hernia, Dyspnoea, Hiatal Hernia, Lung collapse
Fachreza Aryo Damara', Bambang Amam Setya Sulthana ${ }^{2}$

1 Faculty of Medicine, University of Padjadjaran-Dr Hasan Sadikin Hospital

2 Department of Surgery, Faculty of Medicine, University of

Padjadjaran-Dr Hasan Sadikin Hospital

\section{Correspondence to:}

Fachreza Aryo Damara

Faculty of Medicine, University of Padjadjaran-Dr Hasan Sadikin Hospital

fachrezaaryo21@gmail.com 


\section{Introduction}

Diaphragmatic hernia, also known as hiatal hernia, is a protrusion of abdominal organs to the thoracic cavity through the diaphragmatic defect. The defect can be congenital, due to mechanical trauma or idiopathic. There are four types of hiatal hernia based on the grade of abdominal organ protrusion to the thoracic cavity. Type IV hiatal hernia is the least common hernia which occupies less than $5 \%$ of total hiatal hernia cases. ${ }^{1}$ The clinical manifestations of patients with hiatal hernia vary from dysphagia, regurgitation, epigastric pain, postprandial fullness, nausea, emesis and bloating secondary to mechanical obstruction. ${ }^{2}$ Respiratory symptoms may also appear as large abdominal organs may compress the lung.' However, the level of lung compression in the patient is rarely enough to cause atelectasis. ${ }^{3}$

This report describes the case of a 57-yearold man who was referred to the hospital with type IV hiatal hernia. Chest x-ray and $\mathrm{CT}$ imaging were performed and the patient was diagnosed with type IV hiatal hernia with left lung atelectasis. This case represents the atelectasis occurrence secondary to pulmonary compression by abdominal organs that is an extremely rare case in the patient with hiatal hernia. The aim of the study is to provide another clinical significance caused by an excessive compression from herniated abdominal content to thoracic space.

\section{Case History}

A 57-year-old man was referred from a secondary healthcare hospital to the
Department of Surgery at Dr Hasan Sadikin Hospital with complaint of dyspnoea. The patient had dyspnoea since a week prior to being admitted to secondary hospital, and it became progressively worse. The complaint was not associated with activity and changing the environment. No sign of dysphagia, regurgitation, epigastric pain, postprandial fullness, nausea, emesis, and bloating were mentioned by the patient. The patient denied any history of trauma, fever, chronic cough and weight loss. No family history was related to his complaint. Chest tube thoracostomy on the left lung was done at secondary hospital before being referred to Dr Hasan Sadikin Hospital for having diaphragmatic hernia.

On physical examination, the patient's general appearance and vital signs were within normal limits. No abnormalities were found on chest movement, Bowel sound was found positive on the left chest.

A week prior to the admission, the patient had complained of dyspnoea. Then he was brought to secondary hospital because his condition was getting worse. Chest $x$-ray and CT scan examination were performed at a prior hospital and since the results showed signs of diaphragmatic hernia and atelectasis, the patient was then referred to the department of surgery $\mathrm{Dr}$ Hasan Sadikin Hospital. A chest tube thoracostomy was inserted prior to being referred to the hospital.

The patient was diagnosed with diaphragmatic hernia with left lung atelectasis based on symptoms, physical examination and imaging results. He complained of dyspnoea and there were 
bowel sounds on left chest auscultation. In laboratory examination, no significant abnormality was found. However, his oxygen saturation was 93 and there was a slight increase in leukocyte with 13290/ $\mu \mathrm{L}$. Further examination of $x$-ray revealed that major parts of the bowel had moved into the thoracic cavity. (Figure $\mathbf{1}$ )

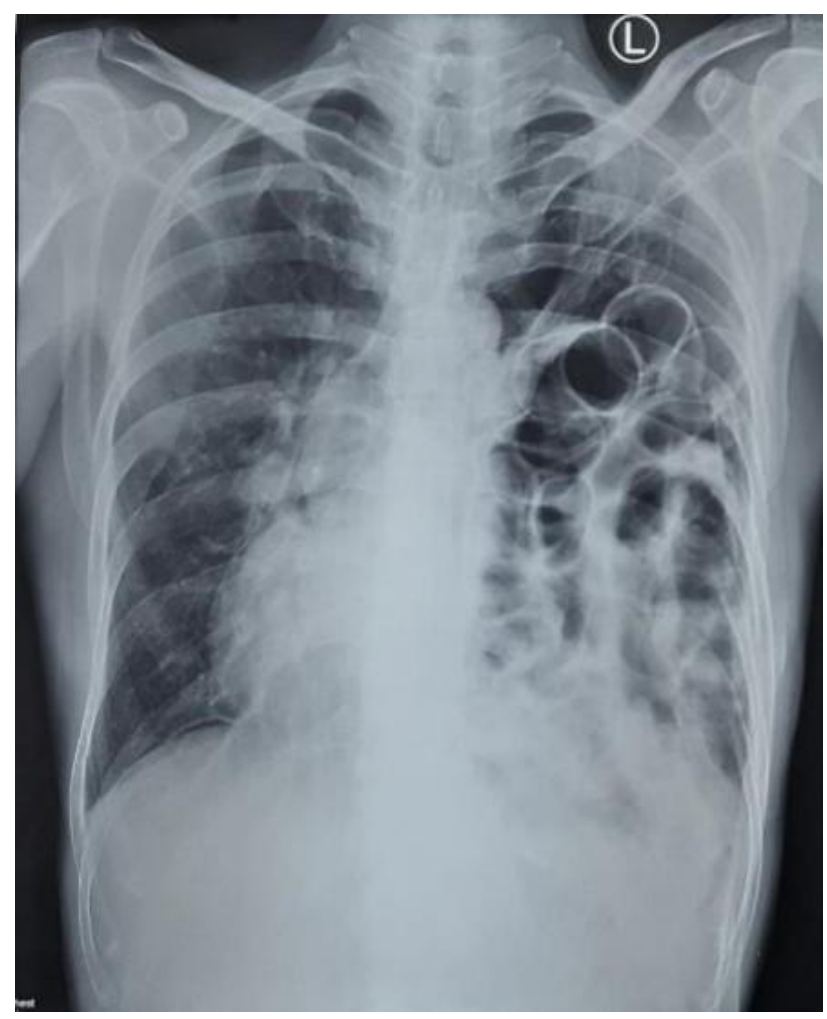

Figure 7 Thorax $x$-ray type IV diaphragmatic hernia

CT scan showed avascular hypodense lesions at left hemithorax with signs of bowel, gastric and faecal material (Figure 2).

Also, on CT scan the left upper thorax showed signs of compressed atelectasis. Therefore, this patient was diagnosed with Type IV Diaphragmatic Hernia with atelectasis.
Open laparotomy was performed. Intraoperatively, clear peritoneal fluid was found flowing from the thoracic cavity. Part of the stomach and jejunum were partially adhered within the thorax cavity. Small bowel was assessed macroscopically, and it was found viable, red-yellow in colour and with normal peristaltic movement. Ultimately, a defect of $10 \mathrm{~cm} \times 10 \mathrm{~cm} \times 3 \mathrm{~cm}$ in size on the left diaphragm with pericardium was seen. The defect was then repaired using polypropylene hernia mesh.

After the repair of the diaphragm, chest tube thoracostomy was performed to further expand the lung. The patient was monitored for his vital signs, blood laboratory examination and regular assessment for the chest tube. There were no abnormalities in vital signs and blood results. Chest tube drain contained serous fluid without signs of infection or inflammation. The patient was treated with a proper solid food diet and fluid therapy.

On day 12 post surgery, no fluid flowed from the chest tube and the patient was stable. The patient recovered well and was discharged in stable condition. He was also advised to follow up his condition to the digestive surgery clinic. After a month of follow up, the patient was doing well based on clinical observation and profile evaluation. 


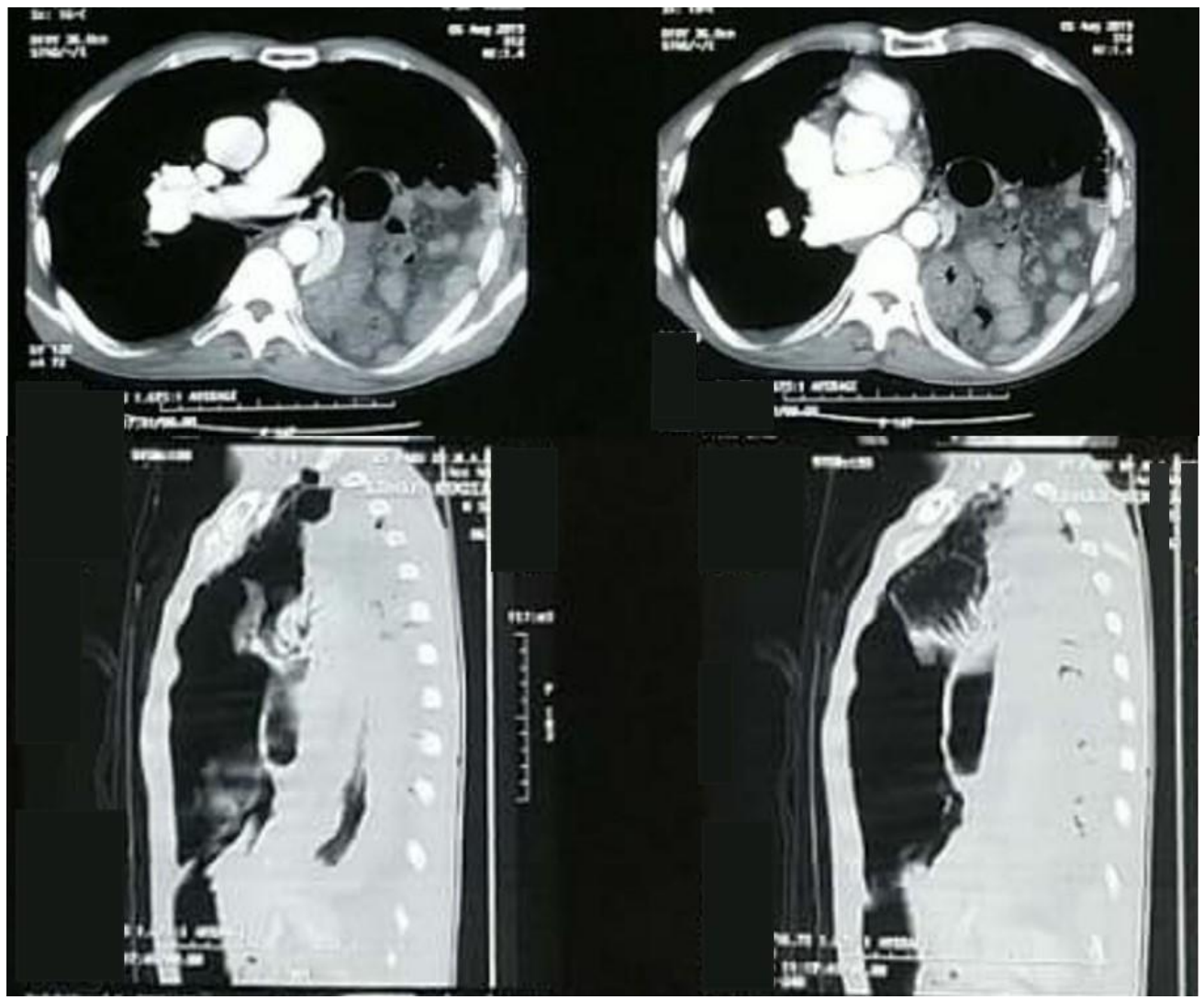

Figure 2 CT scan of thorax showing lung collapse due to compression atelectasis.

\section{Discussion}

To the best of our knowledge, no relevant study reports the occurrence of atelectasis secondary to hiatal hernia. However, a study by Sahin et al revealed the rarely presenting dyspnoea in a patient with large intra-abdominal hiatal hernia. ${ }^{4}$ There was evidence of atelectasis caused by dilated oesophagus in the patient with scleroderma. ${ }^{3}$ Another study reported right lung atelectasis in a patient with achalasia. $^{5}$

Hiatal hernia is the condition where abdominal organs have protruded or moved to the thoracic cavity. Many theories have been established regarding the mechanism of hiatal hernia development. One of the mechanisms in adult hernia is increase in intra-abdominal pressure as it is proved that increased pressure leads to the displacement of the abdominal organs toward the thoracic cavity. ${ }^{6}$ Abdominal organs within the thoracic cavity will compress the lung so the transmural pressure will be reduced leading to compression atelectasis. 7 Hence, the exact pressure and factors that predisposed to compression atelectasis deserve further evaluation.

The patient was diagnosed with left lung atelectasis based on the result of chest $x$ ray imaging and CT-scan results. ${ }^{7,8}$ In the case of atelectasis, bronchoscopy was planned to further evaluate the collapsed lung from intra bronchial view to confirm any material obstructing the airway. However, the patient refused the procedure due to financial reasons. The 
patient was treated operatively with laparoscopic herniorrhaphy using polypropylene hernia mesh. Although the absence of an intraoperative clinical picture becomes our limitation in presenting the case, we accomplished to present the intraoperative condition of the patient with imaging. Also, the surgical approach in this case was similar to other open hiatal hernia repair procedures. Ultimately, the purpose of this report was to present the rare presenting case of hiatal hernia that might add another perspective to the physicians in facing the patient with atelectasis as the result of a high level of lung compression secondary to type IV hiatal hernia.

\section{Declaration of Patient Consent}

The authors certify that they have obtained all appropriate patient verbal consent. The patient has given his consent for his images and other clinical information to be reported in the journal. The patient understands that his name and initials will not be published and their identity will be concealed, but anonymity cannot be guaranteed.

\section{Acknowledgement}

We thank the patient and the family for their contribution to this study.

\section{References}

1. Lebenthal A, Waterford SD, Fisichella PM. Treatment and Controversies in Paraesophageal Hernia Repair. Front Surg. 2015;2(April):1-6.
2. Choi S, Tang A, Murthy S, Raja S. Preoperative Evaluation and Clinical Decision Making for Giant Paraesophageal Hernias: Who Gets an Operation? Thorac Surg Clin. 2019;29(4):415-9.

3. St-Arnaud C, Bouchard N, Lanthier L. Lung Atelectasis Secondary to Massive Esophageal Dilation in a Patient with Scleroderma. Can Respir J. 2010;17(4):e96-8.

4. Sahin C, Akın F, Cullu N, Özseker B, Kirli i, Altun i. A Large Intra-Abdominal Hiatal Hernia as a Rare Cause of Dyspnea. Case Reports Cardiol. 2015;2015(Figure 1):1-4.

5. Liang CY, Lin MS. Achalasia. N Engl J Med. 2009 Feb 19;360(8):801.

6. Yu HX, Han CS, Xue JR, Han ZF, Xin H. Esophageal hiatal hernia: risk, diagnosis and management. Vol. 12, Expert Review of Gastroenterology and Hepatology. Taylor and Francis Ltd; 2018. p. 319-29.

7. Peroni DG, Boner AL. Atelectasis: mechanisms, diagnosis and management. Paediatr Respir Rev. 2000;1(3):274-8.

8. Hubmayr RD. Perspective on lung injury and recruitment: A skeptical look at the opening and collapse story. Am J Respir Crit Care Med. 2002;165(12):1647-53. 